e-mail: rbeaton@princeton.edu

1. Brown, T. M. et al. Astrophys. J. Lett. 856, L6 (2018)

2. Gaia Collaboration. Preprint at https://arXiv.org/ abs/1804.09365 (2018).

3. Bessel, F. W. Mon. Not. R. Astron. Soc. 4, 152-161 (1938)
4. Lindegren, L. et al. Astron. Astrophys. 595, A4 (2016).

5. Reid, M. J. \& Honma, M. Annu. Rev. Astron. Astrophys. 52, 339-372 (2014).

6. Riess, A. G., Casertano, S., Anderson, J., MacKenty, J. \& Filippenko, A. V. Astrophys. J. 785 161 (2014)
7. Casertano, S. et al. Astrophys. J. 825, 11 (2016)

8. Benedict, G. F et al. Publ. Astron. Soc. Pacif. 106 327-336 (1994).

9. Gaia Collaboration. Preprint at https://arXiv.org/ abs/1804.09377 (2018).

10.Gaia Collaboration. Astron. Astrophys. 595, A1 (2016).

\section{Human embryonic stem cells get organized}

An embryo's body plan is established by a structure called the organizer. Evidence of this structure in humans has been lacking, but a stem-cell-based protocol has now enabled researchers to demonstrate its existence. SEE LETTER P.132

\section{OLIVIER POURQUIÉ}

I 1924, Hilde Mangold and Hans Spemann performed what became one of the most famous experiments in developmental biology. They grafted various parts of one grafted region induced unpigmented cells from the host to form an extra embryo, resulting in a 'double embryo' reminiscent of conjoined twins ${ }^{1}$ (Fig. 1a). The duo named the grafted region the organizer, because of its extraordinary ability to organize the host cells around it. But in the almost 100 years since this experiment, technical and ethical difficulties have prevented researchers from demonstrating the presence of an organizer in human embryos. On page 132, Martyn et al. ${ }^{2}$ use stem cells to circumvent these challenges and provide the first experimental description of the human organizer.

To fully understand the importance of the organizer, we must go back to the earliest stages of embryonic development. In vertebrates, the fertilized egg rapidly divides to form a ball of poorly organized cells. At a particular developmental time point, some cells on the surface of this ball become internalized, forming tissues called the endoderm and the mesoderm, which respectively give rise to the gut and to muscles and the skeleton. Other cells remain external and give rise to the skin and the nervous system. This fundamental process of internalization is called gastrulation.

The organizer lies immediately adjacent to the site at which cells become internalized during gastrulation. It gives rise to specific tissues lying along the midline of the embryo, including the notochord - a structure that controls aspects of development of the central nervous system and eventually contributes to the intervertebral discs. An equivalent of the salamander organizer has been found in fish a pigmented salamander embryo onto an unpigmented host embryo, and showed that and birds and in mammals such as rodents ${ }^{3}$. In mammals, the structure that acts as an organizer is called the node because it resembles a knot, and the site of internalization is called the primitive streak.

Unlike salamander embryos, mammals develop in the mother's womb. Accessing and culturing mammalian embryos is therefore difficult. Indeed, it wasn't until 1994 that grafts of a mouse node into a host embryo provided experimental proof of the existence of a structure that has organizer properties in mammals ${ }^{4}$. Although no perfect second embryos were formed in these experiments, the grafted nodes did induce the formation of host-derived neural tissues and sometimes other embryonic tissues.

Human embryos greatly resemble mouse embryos and contain a structure that looks similar to the mouse node ${ }^{4}$. Theoretically, showing that this structure does indeed have the role of an organizer would require researchers to access embryos at three weeks of age (when gastrulation occurs), to graft the node onto a host embryo, and to test whether it induces the formation of a host-derived nervous system and skeletal structures. However, obtaining intact human embryos at this stage, for example from a pregnancy termination, is extremely problematic. Thus, whether the node represents a functional organizer in human embryos has remained unproven.

One alternative would be to let embryos obtained from in vitro fertilization (IVF) develop in culture until the three-week stage, when the node should be present. However, following an ethical consensus that is enshrined in law in many countries, human embryos cannot be cultured in vitro beyond 14 days, making these studies currently impossible.

A second alternative involves the use of pluripotent stem cells, which can give rise to all the body's cell lineages. Protocols to direct in vitro differentiation of these cells make it possible to recapitulate several aspects of

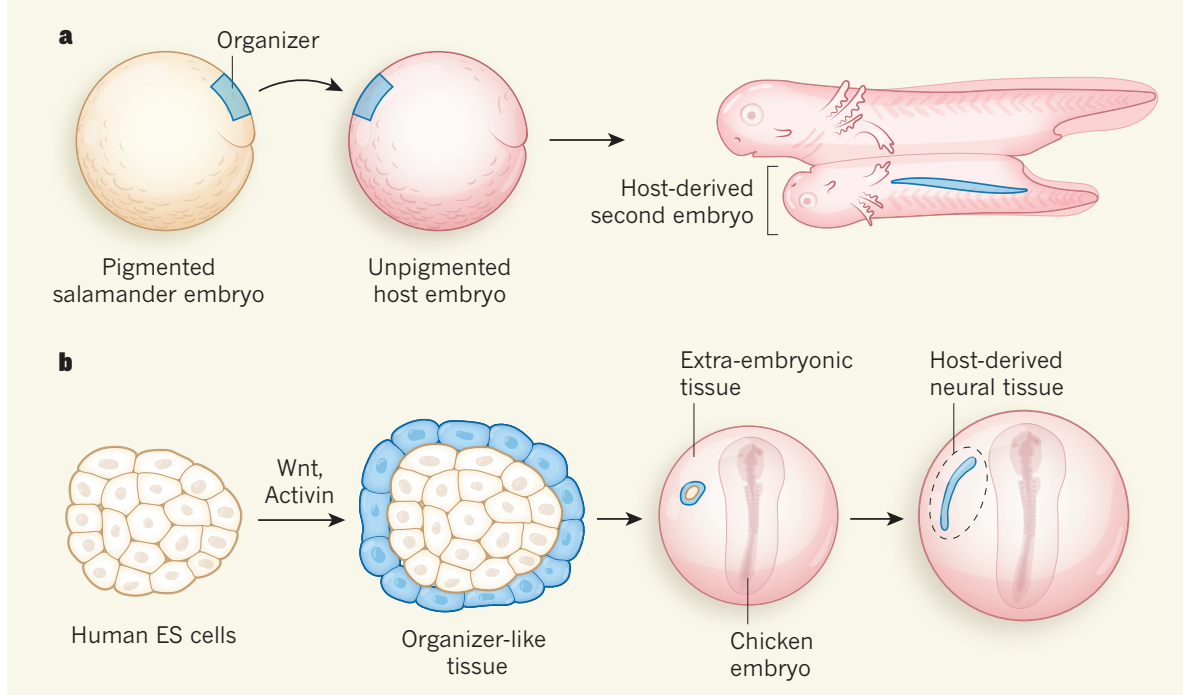

Figure 1 | Experimental demonstration of organizer structures. a, In 1924, an experiment ${ }^{1}$ revealed the properties of an embryonic structure called the organizer. When taken from a pigmented salamander embryo and grafted onto an unpigmented host, the organizer induced the formation of a second embryo derived from unpigmented host cells. b, Martyn et al. ${ }^{2}$ have demonstrated the existence of human cells endowed with similar properties, using human embryonic stem (ES) cells. The authors treated circular discs of ES cells with the growth-factor proteins Wnt and Activin to produce organizer-like cells (blue). When the discs are grafted onto the extra-embryonic tissue around a chick embryo, they induce the host tissue to form an elongated stretch of neural tissue - the standard test for organizer properties. 
embryonic development in a culture dish.

Pluripotent cells derived from human embryos, called embryonic stem (ES) cells, generally form poorly organized colonies when grown in culture. However, the group that performed the current study previously induced ${ }^{5}$ ES cells to self-organize in a way that resembles early embryonic development. They achieved this by culturing the cells on circular micropatterns (microprinted discs of a material called extracellular matrix that is an optimal substrate for the cells) in the presence of the growth factor protein BMP4. The cultures formed endoderm and mesoderm but did not produce primitive streaks or node-like structures.

Martyn et al. took this strategy a step further. They successfully differentiated human ES cells into a node-like tissue by treating their micropatterned cultures with a combination of the growth factors Wnt and Activin, which are crucial for primitive streak and node formation in mice and other vertebrates ${ }^{6,7}$. This treatment led to the formation of a structure that showed characteristics of a primitive streak and to the induction of cells that produced organizerspecific proteins, such as Goosecoid ${ }^{8}$.

To test whether this structure also has the functional characteristics of an organizer, the authors grafted its cells onto chicken embryos, in an area destined to give rise to extra-embryonic tissues that support embryonic development. Remarkably, the grafted cells organized into a notochord-like tissue and induced host cells to form elongated neural tissue (Fig. 1b), demonstrating that the grafted structure has the properties of an organizer.

One could argue that these experiments still raise ethical concerns because they are performed using human ES cells derived from an early-stage human embryo. However, pluripotent cells generated by reprogramming adult cells, which have essentially identical properties to ES cells, could be used as an alternative, alleviating this concern in future studies.

Martyn and colleagues' experimental system provides an alternative to using embryos to study the human embryonic node. Moreover, their experiments suggest that there is striking evolutionary conservation of organizer function from fish to humans. How the organizer organizes the surrounding embryonic tissues into an embryo remains poorly understood, for now at least. But the ability to produce organizer tissue in unlimited amounts in vitro will allow researchers to dissect organizer function at an unprecedented level. .

Olivier Pourquie is in the Department of Genetics, Harvard Medical School, and in the Department of Pathology, Brigham and Women's Hospital, Boston, Massachusetts 02115, USA.

e-mail:pourquie@genetics.med.harvard.edu

1. Spemann, H. \& Mangold, H. Wilhelm Roux Arch.
Entw. Mech. Org. 100, 599-638 (1924).

2. Martyn, I., Kanno, T. Y., Ruzo, A., Siggia, E. D.

\& Brivanlou, A. H. Nature 558, 132-135

(2018).

3. Arias, A. M. \& Steventon, B. Development $\mathbf{1 4 5}$ dev159525 (2018).

4. Beddington, R. S. P. Development $120,613-620$ (1994).

5. Warmflash, A., Sorre, B., Etoc, F., Siggia, E. D. \&
Brivanlou, A. H. Nature Meth. 11, 847-854 (2014).

6. Crease, D. J., Dyson, S. \& Gurdon, J. B. Proc. Natl Acad. Sci. USA 95, 4398-4403 (1998).

7. Gritsman, K., Talbot, W. S. \& Schier, A. F. Development 127, 921-932 (2000).

8. Cho, K. W. Y., Blumberg, B., Steinbeisser, H. \& De Robertis, E. M. Cell 67, 1111-1120 (1991).

This article was published online on 23 May 2018.

\section{CLIMATE CHANGE}

\section{Tropical cyclones are becoming sluggish}

The speed at which tropical cyclones travel has slowed globally in the past seven decades, especially over some coastlines. This effect can compound flooding by increasing regional total rainfall from storms. SEE LETTER P.104

\section{CHRISTINA M. PATRICOLA}

$\mathrm{T}$ Tropical cyclones are among the deadliest and costliest of disasters (go.nature. com/2h59avp), causing destruction not only from strong winds, but also from flooding and mudslides associated with storm surges and heavy rainfall. The total amount of storm rainfall over a given region can be extreme, regardless of the maximum storm wind speeds; it is proportional to the rainfall rate and inversely proportional to the translation speed $^{1}$ (how quickly a tropical cyclone passes over a region). Some studies have investigated trends in heavy rainfall from tropical cyclones over the past century ${ }^{2}$ and future projections in tropical-cyclone rainfall rates ${ }^{3}$, but the translation speed has received less focus. On page 104, Kossin ${ }^{4}$ investigates global trends in tropicalcyclone translation speed, and regional trends over individual ocean basins and adjacent land. He finds that translation speeds have slowed, suggest-

ing that the total amount of regional rainfall from tropical cyclones might have increased.

Kossin analysed 68 years of obser-

"The study finds a10\% global decrease in the speed at which tropical cyclones move." vations made from 1949 to 2016, the longest period for which global data on the locations of tropical cyclones were available. The uncertainty associated with observed trends in translation speed is minimal during this period, because the locations of the tropical cyclones are accurately known. By contrast, it is more difficult to detect trends in the number and intensity of tropical cyclones during this period, because some of these cyclones were not detected in the pre-satellite $\mathrm{era}^{5}$ (before the 1960s). Kossin finds a $10 \%$ global decrease in tropical-cyclone translation speed over this period, a trend that withstands rigorous statistical testing and is dominated by tropical cyclones over the ocean.

The author found that changes in the translation speed of tropical cyclones over land which are more relevant to society than those over the ocean - vary substantially by region. This is unsurprising, because only $10 \%$ of the original data are for such cyclones, and categorizing by region reduces the data sample further, making it more difficult to detect a signal among the noise. Nonetheless, statistically significant slowdowns of $20-30 \%$ have occurred over land regions next to the western North Pacific Ocean, the North Atlantic Ocean and around Australia.

Kossin's work highlights the importance of considering how global-scale atmospheric circulation can influence regional totals of tropical-cyclone rainfall. Tropical cyclones tend to 'go with the flow', meaning that the direction and speed at which they travel are guided by the winds in the surrounding environment. Therefore, any change in tropical circulation could conceivably affect tropical-cyclone translation speed, as Kossin reasons.

One limitation of this study is that it leaves open the question of what is happening to the rate of tropical-cyclone rainfall. The laws of thermodynamics reveal that, as the atmosphere warms by $1^{\circ} \mathrm{C}$, the amount of moisture it can hold increases by $7 \%$. This suggests that global warming can enhance rainfall. However, it is unclear whether there are statistically robust trends in the total amount of regional tropical-cyclone rainfall, or how much the translation-speed slowdowns reported by Kossin could contribute to them. The availability and quality of data pose a challenge to our understanding of rainfall in general - the spatial distribution of rain gauges and radar observations of rainfall vary regionally, and satellite observations are limited to the past few decades and must be analysed using various assumptions to extract rainfall data. However, 